\title{
Rendimiento de la biopsia por aspirado en el diagnóstico del carcinoma de endometrio
}

\author{
Laura Baquedano Mainar ${ }^{1}$, Ignacio Adiego Clavo ${ }^{1}$, Isabel Morollón Loriz ${ }^{1}$, Daniel De \\ Pablo Soriano ${ }^{1}$, Pilar Del Tiempo Marqués ${ }^{1}$, Miguel Angel Ruiz Conde. ${ }^{1}$ \\ ${ }^{1}$ Servicio de Ginecología. Hospital Universitario Miguel Servet. Zaragoza, España.
}

\section{RESUMEN}

Objetivo: Analizar la concordancia histológica pre y postoperatoria del cáncer de endometrio (CE) diagnosticado por cánula de biopsia tipo Cornier. Métodos: Se incluyen 82 pacientes sometidas a biopsia ambulatoria a ciegas por aspirado con diagnóstico anatomo-patológico final de CE en la pieza de histerectomía. Se estudia la concordancia atendiendo al tipo y al grado histológico haciendo dos grupos para cada análisis: CE endometrioide/no endometrioide y CE de bajo/alto grado. Resultados: La sensibilidad de la biopsia para detectar malignidad fue del $100 \%$. El grado de concordancia atendiendo al tipo histológico fue de $94 \%$ (96,7\% para los tipo endometrioide y del $85 \%$ para los tipos no endometrioides). En cuanto al grado histológico la precisión en el diagnóstico fue del 90,2\% (94,3\% en los tumores de bajo grado y $82,7 \%$ en los de alto grado) con una tasa de subestimación del grado tumoral del $6 \%$. Conclusiones: La biopsia por aspiración con cánula tipo Cornier detecta adecuadamente el CE y la concordancia entre la biopsia preoperatoria y la pieza quirúrgica es alta, por lo que puede considerarse una técnica fiable en el diagnóstico del cáncer de endometrio y precisa en la valoración del grado y tipo histológicos. El grupo más discordante es el CE G3 de tipo endometrioide.

\section{PALABRAS CLAVE: Cáncer de endometrio, cánula de Cornier, concordancia histológica}

\section{SUMMARY}

Objective: Analyze pre and post operative histological concordance of endometrial cancer (EC) diagnosed by Cornier biopsy cannula. Methods: 82 patients under going out patient biopsy aspirate blindly with EC in the hysterectomy specimen. We study the type and histological grade accuracy by two groups for each analysis: EC endometrioid/non-endometrioid and low/high grade. Results: The sensitivity for detecting malignancy was $100 \%$. The histological type agreement was $94 \%(96.7 \%$ for the endometrioid type and $85 \%$ for non-endometrioid type). The histological grade accuracy was $90.2 \%(94.3 \%$ in low-grade tumors and $82.7 \%$ in high grade) with a rate of under estimation of tumor grade of $6 \%$. Conclusions: Cornier aspiration biopsy is safe in the diagnosis of EC. Grade and histological type accuracy between preoperative biopsy and surgical specimen is high. The most discordant is EC G3 endometrioid type.

KEY WORDS: Endometrial cancer, Cornier cannula, histological concordance 


\section{INTRODUCCIÓN}

El carcinoma de endometrio (CE) es la neoplasia ginecológica más frecuente en los países desarrollados. En 2012, en España, la incidencia fue de $11,6 / 100.00$ mujeres, con una tasa de mortalidad de 1,88/100.000mujeres (1). La mayoría de los casos son diagnosticados en estadíos iniciales, por eso la tasa de mortalidad es baja y la supervivencia global a 5 años es mayor del $80 \%$. Sin embargo, en las últimas décadas, se advierte un incremento en la incidencia y en la mortalidad por CE. Los cánceres de alto grado, endometrioides pobremente diferenciados, células claras, seroso papilar y carcinosarcomas son un grupo histológico de tumores agresivos que se asocian a altas tasas de recurrencia y son los responsables de la mayoría de las muertes por cáncer de endometrio a pesar de su baja prevalencia (2).

El abordaje quirúrgico en estas pacientes es diferente al de aquellas con tumores bien o moderadamente diferenciados (G1, G2). En todas las pacientes con CE se recomienda como estadiaje quirúrgico la realización de histerectomía total extrafascial, anexectomía bilateral, citologías peritoneales y biopsias de cualquier área sospechosa. Pero mientras que en los casos de tumores G1, G2, con estudio de extensión negativo, la linfadenectomía de territorios pélvicos y paraaórticos se reserva para los casos de infiltración profunda del miometrio, en los tumores de alto grado será imperativa, debiendo realizar también omentectomía en los CE de tipo II. Si en la pieza quirúrgica se obtiene un tumor de alto grado y se ha realizado una cirugía de estadiaje incompleta, se podrá optar por indicar cirugía de reestadiaje (linfadenectomía pélvica y paraaórtica con o sin omentectomía) o administrar radioterapia externa con campos ampliados a territorios pélvicos y para aórtico con o sin quimioterapia. Por ello, resulta imprescindible contar con un diagnóstico histológico prequirúrgico certero para evitar incrementar la morbilidad en estas pacientes, siendo el objetivo fundamental evitar los falsos negativos en el diagnóstico de tumores de alto grado histológico.

El diagnóstico de CE resulta del estudio de una biopsia de endometrio que puede obtenerse a partir de los siguientes procedimientos: legrado uterino, biopsia a ciegas o bajo visión directa histerocópica (3). El legrado uterino fraccionado tiene actualmente más indicaciones terapéuticas que diagnósticas, quedando como opción ante la no obtención de diagnóstico de CE en una paciente con alta sospecha, o cuando las otras opciones diagnósticas han fallado.

Generalmente la biopsia endometrial se realiza de forma ambulatoria, mediante mecanismos de aspirado tipo cánula de Cornier (u otros similares tipo Pipelle, Pipette..) y/o histerocopia. Aunque esta última se considera la prueba de referencia en el diagnóstico de $\mathrm{CE}$, el alto coste que conlleva hace que su disponibilidad sea más limitada de lo deseado y en muchas ocasiones el diagnóstico viene dado por la biopsia a ciegas por aspirado ya que es una técnica más rentable y eficiente. Sin embargo, en ocasiones los especialistas tienen la sensación de que se trata de una técnica que subdiagnostica este tipo de tumores, e indican la realización de histeroscopia diagnóstica ante un primer episodio de sangrado uterino anómalo o el hallazgo de un endometrio engrosado en paciente asintomática, con el aumento de la lista de espera y el gasto que ello supone.

El objetivo del estudio es analizar la concordancia histológica pre y postoperatoria de las pacientes con CE diagnosticadas por biopsia a ciegas por aspirado con cánula tipo Cornier.

\section{PACIENTES Y MÉTODOS}

Estudio retrospectivo, observacional, Iongitudinal, de las pacientes diagnosticadas de CE mediante biopsia a ciegas con cánula tipo Cornier, en el Hospital Universitario Miguel Servet de Zaragoza (HUMS) y tratadas en el mismo centro desde Enero de 2003 a Diciembre de 2013. Se comparó el resultado anatomo-patológico de la biopsia con el definitivo de la pieza de histerectomía. Se seleccionaron las pacientes que cumplían los siguientes criterios de inclusión y ninguno de los de exclusión:

Criterios de inclusión: 1. Pacientes sometidas a biopsia por aspiración de tipo Cornier como método diagnóstico del CE, en las que se obtuvo muestra adecuada para su análisis. 2. Diagnóstico anatomopatológico final en pieza de histerectomía de CE.

Criterios de exclusión: 1. Pacientes a las que se realizó histeroscopia o legrado uterino como método de diagnóstico. 2. Pacientes en las que se practicó biopsia a ciegas por aspirado sin obtener muestra adecuada para su estudio. 3. Pacientes con CE como hallazgo en pieza de histerectomía sin biopsia previa. 4. Pacientes en las que no existe malignidad en la pieza definitiva con biopsia previa positiva para CE. 5. Pacientes con histología de CE en la biopsia que no se intervinieron.

Las biopsias fueron realizadas de forma ambulatoria en las consultas externas del HUMS o de algunos de sus centros médicos de especialidades de referencia. No se indicaba preparación cervical de rutina, sólo cuando se objetivaba a la exploración una estenosis marcada del orificio cervical externo, utilizando misoprostrol a dosis de 200 microgramos de comprimidos vaginales la noche anterior y/o unas 4 horas antes de la técnica. Si además existía atrofia genital marcada, los días previos se administraba estrogenoterapia local.

El manejo de las pacientes con sangrado uterino anómalo que llegaban a consulta se 
realiza siguiendo el protocolo del Servicio. Todas las pacientes dieron su consentimiento para la realización de la técnica.

Las muestras fueron analizadas por patólogos expertos en Ginecología siguiendo la clasificación de la Sociedad Internacional de Patólogos Ginecológicos y la Organización Mundial de la Salud (ISGP/WHO) en cuanto al tipo histológico. Para su análisis estadístico fueron clasificados como endometrioides y no endometrioides. La valoración del grado histológico se realizó siguiendo las recomendaciones de la Federación Internacional de Ginecólogos y Obstetras (FIGO) (G1: El 5\% del tumor está constituido por áreas sólidas no escamosas; G2: Del $6 \%$ al $50 \%$ del tumor está constituido por áreas sólidas no escamosas; G3: Más del $50 \%$ del tumor está constituido por áreas sólidas no escamosas). Para su estudio estadístico se diferenciaron dos grupos: bajo grado (G1, G2) y alto grado (G3).

La recogida de datos se realizó mediante la obtención de las historias clínicas custodiadas por el Servicio de Admisión y Documentación Clínica del HUMS. La información incluyendo las variables del estudio, fue recogida en una base de datos del programa informático SPSS IBM para Windows versión 19.0

Este estudio se realiza de acuerdo con los principios éticos que tienen sus orígenes en la Declaración de Helsinki. Los autores declaran la no existencia de ningún conflicto de intereses.

\section{RESULTADOS}

Desde Enero de 2003 hasta Diciembre de 2013, se diagnosticaron en el Hospital Universitario Miguel
Servet de Zaragoza 550 cánceres de endometrio, de los cuales, 87 fueron diagnosticados mediante biopsia a ciegas ambulatoria con cánula tipo Cornier (15,8\%), que fueron las seleccionadas para el estudio. El resto $(84,2 \%)$ fueron diagnosticados mediante biopsia histerocópica, legrado uterino fraccionado o utilizando varios procedimientos. Se excluyeron 5 pacientes que no fueron intervenidas por no poder en estos casos obtener un resultado anatomo-patológico definitivo. A las 82 pacientes restantes, que fueron las incluidas definitivamente en el estudio, se les realizó cirugía de estadiaje para CE siguiendo las indicaciones de la FIGO.

En las 82 pacientes se detectó malignidad en la biopsia. Es decir la sensibilidad de la técnica para diagnosticar CE fue del $100 \%$.

El resultado del grado y tipo histológico en la biopsia preoperatoria y el estudio definitivo de las pacientes se muestra en la Tabla I. En 58 casos $(70,8 \%)$ el diagnóstico definitivo fue CE endometrioide y los 24 restantes $(29,2 \%)$ CE no endometrioide. La biopsia de endometrio predijo correctamente el tipo histológico del tumor en 77 de las 82 pacientes, lo cual supone una concordancia global del $94 \%$ : $96,7 \%$ para los tipos endometrioide y $85 \%$ para los tipos no endometrioides.

Tuvieron cánceres de alto grado 29 pacientes o G3 en el resultado anatomo-patológico definitivo $(64,6 \%)$ y $53(35,7 \%)$ de bajo grado G1-G2. La sensibilidad para detectar tumor de alto grado fue del $82,7 \%$ y concordancia global entre la biopsia y la pieza de histerectomía en cuanto al grado histológico fue del 90,2\%: 94,3\% en los tumores de bajo grado y $82,7 \%$ en los de alto grado o pobremente diferenciados.

\section{RESULTADO DEL GRADO Y TIPO HISTOLÓGICO EN LA BIOPSIA PREOPERATORIA Y EL ESTUDIO DEFINITIVO}

\begin{tabular}{|c|c|c|c|c|c|c|c|c|c|}
\hline & & & \multicolumn{6}{|c|}{ Resultado definitivo } & \multirow{3}{*}{ Tota } \\
\hline & & & \multicolumn{2}{|c|}{ Bajo grado } & \multicolumn{4}{|c|}{ Alto grado } & \\
\hline & & & G1 & G2 & G3E & $\mathrm{CS}$ & SP & $\mathrm{CC}$ & \\
\hline \multirow{7}{*}{$\begin{array}{l}\text { Resultado } \\
\text { biopsia }\end{array}$} & Bajo grado & G1 & 18 & 12 & 2 & 0 & 0 & 0 & 32 \\
\hline & & G2 & 2 & 18 & 3 & 0 & 0 & 0 & 23 \\
\hline & \multirow{4}{*}{ Alto grado } & G3E & 1 & 2 & 0 & 0 & 0 & 0 & 3 \\
\hline & & CS & 0 & 0 & 0 & 7 & 0 & 0 & 7 \\
\hline & & $\mathrm{SP}$ & 0 & 0 & 0 & 0 & 9 & 0 & 9 \\
\hline & & $\mathrm{CC}$ & 0 & 0 & 0 & 0 & 0 & 8 & 8 \\
\hline & Total & & 21 & 32 & 5 & 7 & 9 & 8 & 82 \\
\hline
\end{tabular}

G3E: CE G3 tipo endometrioide; CS: carcinosarcoma; SP: carcinoma serosopapilar; CC: carcinoma de células claras. 
Cinco pacientes tuvieron un diagnóstico de tumor de bajo grado siendo el resultado final carcinoma de endometrio de alto grado, es decir en el 6\% de las pacientes se subestimó el grado tumoral. En todas ellas se trataba de un CE pobremente diferenciado tipo endometrioide. En 4 de ellas se optó por administrar radioterapia externa adyuvante ampliando los campos a territorios pélvicos y paraaórtico y en la quinta se realizó linfadenectomía de ambos campos en el mismo acto quirúrgico ya que en el análisis intraoperatorio de invasión miometrial informó de afectación mayor al 50\%.

Tres pacientes tuvieron un diagnóstico en la biopsia por aspirado de CE de alto grado resultando de bajo grado en el informe definitivo, por lo que la tasa de sobre estimación del grado tumoral en la biopsia por aspirado fue del 3,6\%. El valor predictivo negativo (VPN) de la biopsia tipo Cornier para los subtipos de cáncer de endometrio de alto grado tumoral fue del $91 \%$ y el valor predictivo positivo (VPP) del 88,8\%. El valor global o eficiencia de la biopsia tipo Cornier para detectar de forma válida los tumores de alto grado histológico fue del $90 \%$.

Se diagnosticó carcinoma de endometrio sobre pólipo en 2 pacientes, ambas con histología de alto grado (carcinoma de células claras). La concordancia entre la biopsia y el estudio definitivo fue total.

\section{DISCUSIÓN}

En nuestro estudio, que incluye 82 pacientes diagnosticadas de CE la sensibilidad de la biopsia por aspirado a ciegas con cánula tipo Cornier para detectar malignidad fue del $100 \%$. La sensibilidad y la especificidad para detectar tumores de alto grado, incluyendo los subtipos no endometrioides, fueron altas $(82,7 \%$ y $90,2 \%)$. Por lo tanto consideramos que se trata de una técnica adecuada para el diagnóstico y la evaluación del CE.

En el cáncer de endometrio la planificación terapéutica variará en función de algunas características entre las que destaca el tipo y sobre todo el grado histológico que es un factor pronóstico bien reconocido en el CE $(2,4)$. Es importante obtener un diagnóstico de CE en la biopsia que sea lo más concordante posible con el informe anatomo-patológico definitivo de la pieza de histerectomía. Sin embargo, los estudios que evalúan la capacidad de detectar los tumores de alto grado histológico en dependencia de la técnica diagnóstica realizada son limitados, y en muchas ocasiones hacen referencia sólo al legrado o se presentan los datos conjuntos entre las distintas formas de biopsia.

La biopsia endometrial ambulatoria a ciegas representa para muchos autores el primer procedimiento en el estudio de la metrorragia. Se trata de un método técnicamente sencillo, rápido y barato, por ello es totalmente accesible en una consulta de ginecología y se contempla en el protocolo de la Sociedad Española de Ginecología y Obstetricia como técnica de inicio en el estudio endometrial (3). Su sensibilidad para detectar malignidad varía según las series. Según un meta-análisis, la sensibilidad y la especificidad para el diagnóstico de hiperplasia o CE fue del $81-99 \%$ y del $98 \%$ respectivamente (5). En nuestro estudio, no hubo ningún caso con biopsia negativa en la que posteriormente se confirmara malignidad. Ello supone que la sensibilidad de la biopsia por aspirado para la detección de CE fue del $100 \%$.

Algunos autores valoran la concordancia pre y postquirúrgica haciendo tres grupos en dependencia de la diferenciación tumoral: G1, G2 y G3 $(6,7)$. Sin embargo consideramos que lo realmente interesante para la práctica clínica es dividirlos en dos grupos: bajo y alto grado histológico. En nuestro estudio la concordancia del grado histológico fue del $94,3 \%$ en los tumores de bajo grado y $82,7 \%$ en los de alto grado. Estos resultados están de acuerdo con estudios que muestran mayor concordancia en los tumores de bajo grado, entre los que destaca un trabajo con un tamaño muestral de casi 2000 pacientes (6) y en discordancia con aquellos en los que la correspondencia en los tumores de alto grado es mayor $(7,8)$.

Es cierto que la biopsia por aspirado a ciegas tiene limitaciones; la no posibilidad de acceder a la cavidad uterina o no obtención de muestra suficiente para el estudio histológico son las más importantes. Por el contrario es barata y accesible. La tasa de muestras de tejido inadecuadas para diagnóstico en pacientes postmenopáusicas se sitúa entre 7 - 22\%, y la imposibilidad de realizar la biopsia por estenosis del orificio cervical externo entre el 1 - $5 \%$ $(9,10)$. En estos casos se tendrá que optar por otra técnica diagnóstica, preferiblemente la realización de la biopsia bajo visión directa histeroscópica. Si no se dispone de ella, la lista de espera es larga y supone una demora importante en el diagnóstico o la paciente sangra de forma importante, también se podrá realizar un legrado uterino con fines diagnóstico así como terapeúticos en el último caso. La concordancia de la biopsia endometrial a ciegas ambulatoria y el legrado uterino es alta entre las dos técnicas $(9,10)$.

EI CE es la patología ginecológica en la que el grado cambia más frecuentementeen el análisis final $(11,12)$. Generalmente y en concordancia con nuestros resultados, suele ser subestimado más que sobreestimado (13). Según las series, el grado histológico que se obtiene de biopsias endometriales puede ser menor que el definitivo sobre la pieza de histerectomía hasta en un $30 \%$ de los casos $(7,11,12)$. Nuestra tasa de subvaloración del grado histológico fue más baja, de $6 \%$. Sin embargo es más alta que otros estudios con tasas 
de subestimación que rondan tan sólo el 1\% (14). Esta diferencia puede deberse a una diferencia a la hora de realizar los cálculos: en los primeros trabajos incluyen como discordantes los tumores G1 en la biopsia que pasan a G2 y en los segundos sólo a los que son G1 en la biopsia y pasan a G3. Consideramos que tanto lo uno como lo otro es una estimación errónea, ya que hoy en día en el manejo del CE se reconocen dos grupos: bajo grado, que incluye $\mathrm{G} 1$ y G2 y alto grado representado por los G3, endometrioides o no. Por ello creemos que lo realmente interesante es saber cuántos tumores de bajo grado en la biopsia pasan a alto grado en el informe definitivo.

El porcentaje de pacientes con sobreestimación del grado tumoral en la biopsia por aspirado fue bajo $(3,6 \%)$, por ello la especificidad de la prueba es alta. Esto quiere decir que si en la biopsia tenemos un tumor de alto grado histológico, muy probablemente el resultado definitivo lo confirmará, por lo que la planificación quirúrgica deberá realizarse acorde a ello desde el principio.

El valor global o la eficiencia de la biopsia por aspirado a ciegas con cánula tipo Cornier que indica la proporción de resultados válidos entre el conjunto de resultados fue del $90 \%$, lo cual consideramos un resultado muy positivo.

Si atendemos al tipo histológico, la concordancia pre y post quirúrgica fue mayor en los CE endometrioides que en los no endometrioides o tipo II: $96,7 \%$ en comparación con $85 \%$. A diferencia que en los CE de bajo grado que suelen asentarse en endometrios engrosados e hiperplásicos, los CE de tipo no endometrioide coinciden en muchas ocasiones con endometrios atróficos o coexiten con pólipos. Por este motivo se puede complicar la obtención de una muestra adecuada en la biopsia a ciegas, o aún en caso de poder conseguir material suficiente para su análisis repercuta directa y negativamente en la concordancia con el resultado definitivo (15). Sin embargo, en nuestro estudio todos los CE tipo II o no endometrioides se diagnosticaron correctamente y la dificultad la muestran los CE G3 tipo endometrioide con un 100\% de discordancia. Parece que existe un problema a la hora de estimar las áreas sólidas no escamosas del tumor. Sin embargo, la escasa muestra de CE G3 endometrioides $(n=5)$ no nos permite extraer conclusiones definitivas. Sería interesante que se siguiera investigando en este sentido.

Otras posibles limitaciones en nuestro estudio son el número de pacientes $(n=82)$, que no es muy alto y la inclusión de pocos casos con histología de CE tipo II o no endometrioide $(n=24)$ y de pocos casos de CE sobre pólipo $(n=2)$.

\section{CONCLUSIÓN}

La capacidad de la biopsia por aspiración con cánula tipo Cornier para detectar CE es total y la concordancia entre la biopsia preoperatoria y la pieza quirúrgica es alta, por lo que puede considerarse una técnica confiable en el diagnóstico del cáncer de endometrio y precisa en la valoración del grado y tipo histológico. El mayor grado de discordancia lo compone el grupo de CE G3 de tipo endometrioide.

\section{REFERENCIAS}

1. Ferlay J, Shin HR, Bray F, et al. GLOBOCAN 2008, Cancer Incidence and Mortality Worldwide: IARC Cancer Base No. 10 [Internet]. Lyon, France: International Agency for Research on Cancer; 2010. Disponible en: http://globocan.iarc.fr. Acceso: 20 de mayo de 2013.

2. Ayeni TA, Bakkum-Gamez JN, Mariani A, et al. Comparative outcomes assessment of uterine grade 3 endometrioid, serous, and clear cell carcinomas. Gynecol Oncol 2013;129(3):478-85.

3. Oncoguía SEGO. Cáncer de endometrio 2010. Guías de práctica clínica en cáncer ginecológico y mamario. Publicaciones SEGO, Octubre 2010.

4. Ayhan A, Taskiran C, Yuce K, Kucukali T. The prognostic value of nuclear grading and the revised FIGO grading of endometrial adenocarcinoma. Int J Gynecol Pathol 2003;22(1):71-4.

5. Dijkhuizen FP, Mol BW, Brölmann HA, Heintz AP. The accuracy of endometrial sampling in the diagnosis of patients with endometrial carcinoma and hyperplasia: a meta-analysis. Cancer 2000; 89(8):1765-72.

6. Francis JA, Weir MM, Ettler HC, et al. Should preoperative pathology be used to select patients for surgical staging in endometrial cancer? Int J Gynecol Cancer 2009;19(3):380-4.

7. Wang XY, Pan ZM, Chen XD, Lü WG, Xie X. Accuracy of tumor grade by preoperative curettage and associated clinic pathologic factors in clinical stage I endometriod adenocarcinoma. Chin Med J 2009;122(16):1843-6.

8. Mitchard J, Hirschowitz L. Concordance of FIGO grade of endometrial adenocarcinomas in biopsy and hysterectomy specimens. Histopathology 2003;42(4):3728.

9. Leng X, Wang $\mathrm{M}$, Zhang SL, et al. Different methods for the diagnosis of endometrial histological comparative study. Zhonghua $\mathrm{Fu}$ Chan KeZaZhi 2013;48(12):891-5.

10. Demirkiran F, Yavuz E, Erenel $\mathrm{H}$, et al. Which is the best technique for endometrial sampling? Aspiration (pipelle) versus dilatation and curettage (D\&C). Arch Gynecol Obstet 2012;286(5):1277-82. 
11. Frumovitz M, Singh DK, Meyer L, et al. Predictors of final histology in patients with endometrial cancer. Gynecol Oncol 2004;95(3):463-8.

12. Leitao MM Jr, Kehoe S, Barakat RR, et al. Comparison of D\&C and office endometrial biopsy accuracy in patients with FIGO grade 1 endometrial adenocarcinoma.Gynecol Oncol 2009;113(1):105-8.

13. Huang GS, Gebb JS, Einstein MH, et al. Accuracy of preoperative endometrial sampling for the detection of high-grade endometrial tumors. Am J Obstet Gynecol 2007;196(3):243.e1-5.
14. Leitao MM Jr, Kehoe S, Barakat RR, et al. Accuracy of preoperative endometrial sampling diagnosis of FIGO grade 1 endometrial adenocarcinoma. Gynecol Oncol 2008;111(2):244-8.

15. Guido RS, Kanbour-Shakir A, Rulin MC, Christopherson WA. Pipelle endometrial sampling: sensitivity in the detection of endometrial cancer. J Reprod Med 1995;40:553-5. 